\title{
A Topological Characterization of the Underlying Spaces of Complete R-Trees
}

\author{
PAUL FABEL
}

\begin{abstract}
We prove that a topological space $(P, \tau)$ admits a compatible metric $d$ such that $(P, d)$ is a complete R-tree if and only if $P$ is a topological R-tree (i.e. metrizable, locally path-connected, and uniquely arcwise connected) and also locally interval compact. The latter notion means that each point $x \in P$ has a closed neighborhood $\bar{U}$ such that $\bar{U} \cap \alpha$ is compact for each closed half interval $\alpha \subset P$. For topological R-trees, the property "locally interval compact" is strictly stronger than topological completeness.
\end{abstract}

\section{Introduction}

An $R$-tree $(P, d)$ is a uniquely arcwise connected metric space such that for each pair of points $\{x, y\} \subset P$, the arc $([x, y], d) \subset P$ from $x$ to $y$ is isometric to the Euclidean segment $[0, d(x, y)]$. R-trees have received considerable attention as objects of study in their own right, and R-trees also play a prominent role in geometric group theory, notably in the study of group actions on spaces of nonpositive curvature $[1 ; 2 ; 3 ; 4 ; 5 ; 6 ; 7 ; 8 ; 9 ; 10 ; 11 ; 12 ; 14 ; 15 ; 16 ; 17 ; 18 ; 20$; $21 ; 22 ; 23 ; 24 ; 25 ; 26 ; 29 ; 30]$.

However, the following fundamental question has apparently escaped collective inquiry: Which topological spaces $(P, \tau)$ underly the complete R-trees?

To answer this question, observe that open metric balls in the metric R-tree $(P, d)$ are path connected and hence $(P, \tau)$ is metrizable, uniquely arcwise connected, and locally path connected, that is, R-trees are topological $R$-trees. Thanks to a result of John Mayer and Lex Oversteegen [27], the converse is also true: each topological R-tree $(P, \tau)$ is the underlying space of some R-tree $(P, d)$. (A preprint of the author contains an alternate shorter proof [13].)

For the metric R-tree $(P, d)$ to be complete, it is of course necessary that $(P, \tau)$ is topologically complete, but somewhat surprisingly, this is not sufficient. Example 1, the planar subspace $([0,1] \times\{0\}) \cup\left(\bigcup_{n=1}^{\infty}\left\{\frac{1}{n}\right\} \times\left[0, \frac{1}{n}\right)\right)$, shows it is false that a topologically complete topological R-tree $(P, \tau)$ is necessarily the underlying space of some complete R-tree $(P, d)$.

As mentioned in the abstract, to strengthen topological completeness and ensure that the topological R-tree $(P, \tau)$ is the underlying space of a complete metric $\mathrm{R}$-tree, it is precisely adequate to demand that $(P, \tau)$ has the following extra property:

Received September 29, 2014. Revision received August 8, 2015. 
Definition 1. The space $(P, \tau)$ is locally interval compact if for each $x \in P$, there exists an open set $U \subset P$ such that $x \in U$ and $\alpha \cap \bar{U}$ is compact for all closed subspaces $\alpha \subset P$ such that $\alpha$ is homeomorphic to $[0,1)$.

We also establish that a metric R-tree $(P, d)$ is locally interval compact if and only if $(P, d)$ is open in its metric completion, and in turn such spaces precisely underly complete R-trees. With the exception of the reference to [27], this paper is self contained, and the main result is the following.

Theorem 1. Suppose $(P, \tau)$ is a topological space. The following are equivalent:

(1) There exists a compatible metric $d$ such that $(P, d)$ is a complete $R$-tree.

(2) There exists a compatible metric $d$ such that $(P, d)$ is an $R$-tree and such that $(P, d)$ is an open subspace of its metric completion $\overline{(P, d)}$.

(3) $P$ is metrizable, locally path connected, uniquely arcwise connected, and locally interval compact.

\section{Preliminaries, Examples, Remarks, and Lemmas}

An arc is a single point or a space homeomorphic to $[0,1]$. A $p$-based topological R-tree $\left(P, \tau, p, \leq^{\wedge}\right)$ is a metrizable, uniquely arcwise connected, locally path connected space with $p \in P$ and $[x, y] \subset P$ denoting the unique arc from $x$ to $y$. The space $P$ enjoys both the associative binary operation ${ }^{\wedge}$ such that $\left[p, x^{\wedge} y\right]=$ $[p, x] \cap[p, y]$ and the partial order $\leq$ such that $y \leq x$ iff $y \in[p, x]$. Notationally, we may suppress $\leq$ and $^{\wedge}$ if it is understood that $p$ is the basepoint, and $\tau$ can be replaced by $d$ or $D$ if $P$ is equipped with the particular metric $d$ or $D$. A metric space $(P, d)$ is complete if each Cauchy sequence has a limit, and we remind the reader that every metric space can be embedded as a dense subspace of a complete metric space [28], uniquely up to isometry.

Example 1 . Let $P$ denote the planar subspace $([0,1] \times\{0\}) \cup\left(\bigcup_{n=1}^{\infty}\left\{\frac{1}{n}\right\} \times\right.$ $\left.\left[0, \frac{1}{n}\right)\right)$. Note that $P$ is not the underlying space of a complete R-tree since the half open intervals $\left\{\frac{1}{n}\right\} \times\left[0, \frac{1}{n}\right.$ ) would be forced to have infinite geometric length, violating the topological fact that $x_{n} \rightarrow 0$ if $x_{n} \in\left\{\frac{1}{n}\right\} \times\left[0, \frac{1}{n}\right.$ ). Note that $P$ is a $G_{\delta}$ subspace of the plane (the intersection of countably many open planar sets), and hence $P$ is topologically complete.

The following fact follows easily from the algebraic properties of $\left(P,{ }^{\wedge}, \leq\right)$.

Lemma 1. Suppose $\left(P, p, \tau \leq,^{\wedge}\right)$ is a p-based topological $R$-tree and $[p, z] \cap$ $[x, y]=\emptyset$. Then $x^{\wedge} z=y^{\wedge} z$.

Proof. Note that $a^{\wedge} b \leq b$ since $a^{\wedge} b \in[p, b]$ and $a \leq b \Rightarrow a^{\wedge} b=a$ since $[p, a] \cap$ $[p, b]=[p, a]=\left[p, a^{\wedge} b\right]$. Note $\left\{x^{\wedge} z, x^{\wedge} y\right\} \subset[p, x]$ and $x^{\wedge} z<x^{\wedge} y$ (since otherwise we obtain the contradiction $\left.x^{\wedge} z \in[p, z] \cap\left[x^{\wedge} y, x\right] \subset[p, z] \cap[x, y]\right)$. By a symmetric argument we conclude $y^{\wedge} z<y^{\wedge} x$. Thus, $\left\{x^{\wedge} z, y^{\wedge} z\right\} \subset\left[p, x^{\wedge} y\right]$. 
Note that $y^{\wedge} z \in[p, x] \cap[p, z]$ and thus $y^{\wedge} z \leq x^{\wedge} z$. By a symmetric argument, $x^{\wedge} z \leq y^{\wedge} z$, and thus $x \wedge z=y^{\wedge} z$.

The following lemma is also a consequence of the fact that the metric completion of an R-tree is an R-tree [19;8].

Lemma 2. Suppose $\left(P, d, p, \leq{ }^{\wedge}\right)$ is an incomplete p-based R-tree with metric completion $\overline{\left(P, d, p, \leq{ }^{\wedge}\right)}$. Suppose $y \in \partial P=\overline{(P, d)} \backslash P$. There exists an orderpreserving isometric embedding $h:[0, d(x, y)) \rightarrow(P, d)$ such that $h(0)=p$ and $y=\lim _{t \rightarrow d(x, y)} h(t)$. In particular, since the compactum $h([0, d(x, y)])$ is closed in the metric space $\overline{(P, d)}, h([0, d(x, y))=P \cap h([0, d(x, y)])$ is a closed subspace of $P$.

Proof. Obtain a sequence $z_{n} \in P$ with $d\left(z_{n}, y\right) \rightarrow 0$. For each $N \in\{1,2,3, \ldots\}$, obtain $M_{N}>N$ such that $\left[p, z_{N}\right] \cap\left[z_{m}, z_{n}\right]=\emptyset$ if $M_{N} \leq m \leq n$. Define $y_{N}=z_{N} \hat{\wedge} z_{M_{N}}$ and note that by Lemma $1 y_{N}=z_{N} \hat{\wedge} z_{m} \hat{\wedge} z_{n}=z_{N} \hat{\wedge} z_{m}$ if $M_{N} \leq$ $m \leq n$. Note that $y_{n} \rightarrow y$ and by construction there exists a subsequence $y_{k_{1}}<$ $y_{k_{2}} \ldots$ Let $h:[0, d(x, y)) \rightarrow \bigcup_{k=1}^{\infty}\left[p, y_{n_{k}}\right] \subset P$ be the natural isometry mapping $\left[d\left(p, y_{k_{n}}\right), d\left(p, y_{k_{n+1}}\right)\right]$ onto $\left[y_{k_{n}}, y_{k_{n+1}}\right] \subset P$. By construction, $h$ is continuously extendable at $d(p, y)$.

The following lemma establishes that locally interval compact R-trees are open subspaces of their metric completions.

Lemma 3. Suppose that $(P, d, p)$ is a p-based incomplete $R$-tree and $\partial P=$ $\overline{(P, d, p)} \backslash P$ is not a closed subspace of the metric completion $\overline{(P, d, p)}$. Then $P$ is not locally interval compact.

Proof. Obtain $x \in P \cap \overline{\partial P}$. Suppose $\varepsilon>0$. Obtain $y \in \partial P$ such that $d(x, y)<\varepsilon$. Obtain by Lemma 2 an isometric embedding $[0, d(p, y)] \rightarrow \bar{P}$ such that $0 \mapsto p$, $d(p, y) \mapsto y$, and $[0, d(x, y))$ is order isometric to a closed subspace $\alpha \subset P$. Let $\delta=\varepsilon-d(x, y)$. Obtain $z \in \alpha$ with $d(z, y)<\delta$. Note that if $z<w$ and $w \in \alpha$, then $d(w, x)=d(w, z)+d(z, x)<(\varepsilon-d(x, y))+d(x, y)<\varepsilon$. Thus, $[z, y)$ is a closed subspace of $P,[z, y)$ is homeomorphic to $[0,1),[z, y) \subset \overline{B(x, \varepsilon)}$, and $[z, y)$ is not compact.

Remark 1. If $(P, d, p)$ is a $p$-based $\mathrm{R}$ tree and $\alpha \subset P$ is homeomorphic to $[0,1)$, then $(\alpha, d)$ is isometric to a unique finite Euclidean half open interval $[0, R)$ for some $R>0$ or the infinite ray [0, $)$. If $\alpha$ is closed in $P$ and $(\alpha, d)$ is isometric to the finite interval $[0, R)$, then the preimage of the sequence $R-\frac{1}{n}$ shows that $(P, d, p)$ is incomplete.

The following easy lemma is used in the proof of Lemma 6.

Lemma 4. Suppose that $(X, D)$ is a metric space and $A \subset X$ and $2^{X}$ denotes the collection of compact subsets of $X$ with the Hausdorff distance. Define $L: 2^{X} \rightarrow$ 
$[0, \infty)$ as $L(C)=\inf _{(c, a) \in C \times A} D(c, a)$. Then $L$ is continuous. If $\left(P, d, p, \leq,^{\wedge}\right)$ is an R-tree, then $\lambda$ is continuous if $\lambda: P \rightarrow 2^{P}$ is defined as $\lambda(x)=[p, x]$.

Proof. By definition the Hausdorff distance $H(C, B)$ [28] between compacta $\{B, C\} \subset X$ satisfies $0 \leq H(B, C)<\varepsilon$ iff for each $b \in B$, there exists $c \in C$ with $D(b, c)<\varepsilon$ and for each $c \in C$, there exists $b \in B$ with $D(b, c)<\varepsilon$. If $b \in B$ and $c \in C$ with $D(b, c)<\varepsilon$, then $|L(C)-L(B)|<\varepsilon$, and in particular $L$ is continuous. If $\{x, y\} \subset P$ with $d(x, y)<\varepsilon$, then $H([p, x],[p, y])=d(x, y)<\varepsilon$, and in particular $\lambda$ is continuous.

The following lemma and its proof also appear in another preprint of the author [13].

Lemma 5. Suppose that $\left(P, p, \tau, \leq,^{\wedge}\right)$ is a p-based topological R-tree. Suppose that the continuous function $l: P \rightarrow[0, \infty)$ satisfies $x<y \Rightarrow l(x)<l(y)$. Define $d: P \times P \rightarrow[0, \infty)$ as $d(x, y)=l(x)+l(y)-2 l\left(x^{\wedge} y\right)$. Then $d$ is a metric on the set $P$, inclusion $\kappa:(P, \tau) \rightarrow(P, d)$ is a continuous bijection, each arc $\kappa[x, y] \subset$ $(P, d)$ is isometric to the Euclidean segment $[0, d(x, y)]$, and $d\left(x, x^{\wedge} x_{m}\right) \rightarrow 0 \Rightarrow$ $x^{\wedge} x_{m} \rightarrow x$ in $(P, \tau)$.

Proof. Note that $d(x, x)=0$ since $x^{\wedge} x=x$ and $y \neq x \Rightarrow x^{\wedge} y<x$ or $x^{\wedge} y<y$ and hence $d(x, y)>0 . d(x, y)=d(y, x)$ since $x^{\wedge} y=y^{\wedge} x$. Note that $0 \leq$ $2\left(l(y)-l\left(x^{\wedge} y\right)\right)$ since $x^{\wedge} y \leq y$. Note that $d(x, z) \leq d(x, y)+d(y, z)$ iff $-2 l\left(x^{\wedge} z\right) \leq 2 l(y)-2 l\left(x^{\wedge} y\right)-2 l\left(x^{\wedge} z\right)$ iff $0 \leq 2\left(l(y)-l\left(x^{\wedge} y\right)\right)$. The latter holds since $x^{\wedge} y \leq y$. Thus, $d$ is a metric on the set $P$.

If $x_{m} \rightarrow x$ in $(P, \tau)$, then $x^{\wedge} x_{m} \rightarrow x$ in $(P, \tau)$. Thus, since $l$ is continuous at $x, l(x)-l\left(x_{m}\right) \rightarrow 0$ and $l(x)-l\left(x^{\wedge} x_{m}\right) \rightarrow 0$. Hence, $\left(l(x)-l\left(x^{\wedge} x_{m}\right)\right)+\left(l\left(x_{m}\right)-\right.$ $l(x))+\left(l(x)-l\left(x^{\wedge} x_{m}\right)\right)=d\left(x, x_{m}\right) \rightarrow 0$.

Note that if $\{w, z\} \subset(P, \tau)$ then $w \leq z$ iff $w=z^{\wedge} w$, and hence by definition, $d(w, z)=l(z)-l(w)$. Thus, if $\{x, y\} \subset(P, \tau)$, then the natural homeomorphism $h_{x, y}: \kappa\left[x^{\wedge} y, x\right] \rightarrow\left[0, l(x)-l\left(x^{\wedge} y\right)\right]\left(\right.$ defined as $\left.h_{x, y}(z)=l(z)-l\left(x^{\wedge} y\right)\right)$ is an isometry onto the Euclidean segment since $w<u<z \Rightarrow d(z, w)=l(z)-l(w)=$ $(l(z)-l(u))+(l(u)-l(w))=d(z, u)+d(u, z)$. Pasting at $0\left(h_{y, x}^{-1}\right.$ union the reverse of $\left.h_{x, y}^{-1}\right)$ yields the natural isometry $\left[l\left(x^{\wedge} y\right)-l(x), l(y)-l\left(x^{\wedge} y\right)\right] \rightarrow$ $\kappa[x, y]$.

Suppose $d\left(x, x^{\wedge} x_{m}\right) \rightarrow 0$. Then $\left\{x^{\wedge} x_{m}\right\}$ is a sequence in the (metrizable) compact arc $[p, x] \subset(P, \tau)$. Since $\kappa$ is continuous at $y$, if $y \in[p, x] \subset(P, \tau)$ is a subsequential limit of $\left\{x^{\wedge} x_{m}\right\}$, then $y=\kappa(y)=x$. Hence, $x^{\wedge} x_{m} \rightarrow x$ in $(P, \tau)$.

The standard fact that a space $U$ is topologically complete if $U$ is an open subspace of some complete metric space $(X, d)$ is often established [28] via a closed embedding $\phi: U \rightarrow X \times R$ with $u \mapsto\left(u, \frac{1}{\partial(u, \partial U)}\right)$. For several reasons, this proof does not work "off the shelf" when trying to obtain a complete R-tree metric for a connected open subspace $P \subset Q$ of a complete R-tree $(Q, D)$. Instead, we build a strictly increasing length function $l: P \rightarrow[0, \infty)$ such that $l\left(x_{n}\right) \rightarrow \infty$ if 
$x_{n} \rightarrow \partial P$, apply Lemma 5 , and verify completeness of the metric and continuity of the inverse mapping.

Lemma 6. Suppose that $(Q, D)$ is a complete metric space, suppose that the subspace $P \subset Q$ is open, nonempty, and dense, and suppose that he metric space $\left(P, D, p, \leq{ }^{\wedge}\right)$ is a p-based $R$-tree. There exists a topologically compatible metric $d$ on $P$ such that $(P, d, p)$ is a complete $R$-tree.

Proof. Let $\partial P=Q \backslash P$. Define $L: P \rightarrow[0, \infty)$ as $L(x)=\inf \{D(y, z) \mid y \in$ $[p, x]$ and $z \in \partial P\}$. Note that $L>0$ since $[p, x]$ is compact and $\partial P$ is closed. Note that $y \leq x \Rightarrow L(y) \geq L(x)$ since $[p, y] \subset[p, x]$. Define $l: P \rightarrow[0, \infty)$ as $l(x)=D(p, x)+\frac{1}{L(x)}$. Note that $l$ is continuous since $D$ is continuous and by Remark $4 L$ is continuous. Observe that $\{x, y\} \subset P$ and $x<y \Rightarrow D(p, x)<D(p, y)$ (since $(P, D)$ is an R-tree) and $\frac{1}{L(x)} \leq \frac{1}{L(y)}$ since $L(y) \geq L(x)$, and hence $l(x)<l(y)$. Thus, applying Lemma 5 , the metric $d(x, y)=l(x)+l(y)-2 l\left(x^{\wedge} y\right)$ ensures that the inclusion $\kappa:(P, D) \rightarrow(P, d)$ is a continuous bijection, and $\kappa[x, y] \subset(P, d)$ is isometric to the Euclidean segment $[0, d(x, y)]$. By definition, $D(x, y)=d(x, y)-l(x)-l(y) \leq d(x, y)$. Hence, $\kappa$ is a homeomorphism. Thus, $(P, d)$ is uniquely arcwise connected, and hence $(P, d)$ is an R-tree.

Observe that for real numbers, if $0<t<s$, then $1<\frac{1}{t}-\frac{1}{s}$ iff $s t<s-t$.

To obtain a contradiction, suppose that $(P, d)$ is incomplete. Let $\overline{(P, d)}$ denote the metric completion of $(P, d)$. By Lemma 2 obtain $y \in \overline{(P, d)} \backslash P$, and an isometric embedding $h:[0, d(p, y)] \rightarrow \overline{(P, d)}$, so that $h(0)=p, h(d(p, y))=y$ and $h \mid[0, d(p, y))$ is an order-preserving embedding into $P$. Let $y_{m}=h\left(\frac{d(p, y) m}{m+1}\right)$. Note that $\left\{y_{m}\right\}$ is Cauchy in $(P, d)$ and hence $\left\{y_{m}\right\}$ is Cauchy in $(P, D)$ since $D \leq d$.

Note that for all $m \geq 1$ and $k \geq 1,0<L\left(y_{m}\right) \leq D\left(y_{m}, y_{m+k}\right)$ since $\left[p, y_{m}\right] \subset$ $\left[p, y_{m+k}\right]$. Thus, since $\left\{y_{m}\right\}$ is Cauchy in $(P, D)$, the sequence $L\left(y_{m}\right) \rightarrow 0$. Hence (applying the continuity of $\times: R \times R \rightarrow R$ and $-: R \times R \rightarrow R$ (familiar multiplication and substraction of real numbers)), for each $M \geq 1$, we obtain $N_{M}>M$ so that $L\left(y_{M}\right) \times L\left(y_{n}\right)<L\left(y_{M}\right)-L\left(y_{n}\right)$. Thus, if $n \geq N_{M}>M$, then $y_{M}=y_{M} \hat{\wedge} y_{n}$, and hence $d\left(y_{n}, y_{M}\right)=D\left(y_{n}, y_{M}\right)+\left(\frac{1}{L\left(y_{n}\right)}-\frac{1}{L\left(y_{M}\right)}\right) \geq\left(\frac{1}{L\left(y_{n}\right)}-\frac{1}{L\left(y_{M}\right)}\right)>1$, contradicting the fact that $\left\{y_{m}\right\}$ is Cauchy in $(P, d)$.

\section{Proof of Theorem 1}

For $3 \Rightarrow 2$, suppose that $(P, \tau)$ is a locally interval complete topological R-tree. Obtain by [27] a topologically compatible metric $d$ such that $(P, d)$ is an R-tree. If $(P, d)=\overline{(P, d)}$, then note that $\overline{(P, d)}$ is open in $\overline{(P, d)}$. If $(P, d) \neq \overline{(P, d)}$, then Lemma 3 ensures that $P$ is open in $\overline{(P, d)}$. For $2 \Rightarrow 1$, suppose that $(P, d)$ is an Rtree, open in its metric completion $\overline{(P, d)}$. Apply Lemma 6 . For $1 \Rightarrow 3$, suppose that $(P, d)$ is a complete R-tree. Note that, by definition, $(P, d)$ is metrizable and uniquely arcwise connected, and $(P, d)$ is locally path connected since open metric balls are path-connected. Recall Remark 1 and observe that the bounded open metric balls of radius 1 establish that $(P, d)$ is locally interval compact. 


\section{References}

[1] A. G. Aksoy and M. A. Khamsi, A selection theorem in metric trees, Proc. Amer. Math. Soc. 134 (2006), no. 10, 2957-2966.

[2] J. A. Behrstock, Asymptotic geometry of the mapping class group and Teichmüller space, Geom. Topol. 10 (2006), 1523-1578.

[3] V. N. Berestovskii and C. P. Plaut, Covering R-trees, R-free groups, and dendrites, Adv. Math. 224 (2010), no. 5, 1765-1783.

[4] M. Bestvina, R-trees in topology, geometry, and group theory, Handbook of geometric topology, pp. 55-91, North-Holland, Amsterdam, 2002.

[5] B. H. Bowditch and J. Crisp, Archimedean actions on median pretrees, Math. Proc. Cambridge Philos. Soc. 130 (2001), no. 3, 383-400.

[6] M. R. Bridson and A. Haefliger, Metric spaces of non-positive curvature, SpringerVerlag, Berlin, 1999.

[7] J. W. Cannon, The theory of negatively curved spaces and groups, Ergodic theory, symbolic dynamics, and hyperbolic spaces, Oxford Sci. Publ., pp. 315-369, Oxford Univ. Press, New York, 1991.

[8] I. Chiswell, Introduction to $\Lambda$-trees, World Scientific Publishing Co., Inc., River Edge, NJ, 2001.

[9] I. Chiswell, T. W. Müller, and J.-C. Schlage-Puchta, Completeness and compactness criteria for $R$-trees, preprint.

[10] J. J. Dijkstra and K. I. S. Valkenburg, The instability of nonseparable complete Erdôs spaces and representations in R-trees, Fund. Math. 207 (2010), no. 3, 197-210.

[11] A. Dranishnikov and M. Zarichnyi, Universal spaces for asymptotic dimension, Topology Appl. 140 (2004), no. 2-3, 203-225.

[12] C. Drutu and M. V. Sapir, Groups acting on tree-graded spaces and splittings of relatively hyperbolic groups, Adv. Math. 217 (2008), no. 3, 1313-1367.

[13] P. Fabel, A short proof characterizing topologically the underlying spaces of R-trees, preprint.

[14] H. Fischer and A. Zastrow, Combinatorial R-trees as generalized Cayley graphs for fundamental groups of one-dimensional spaces, Geom. Dedicata 163 (2013), 19-43.

[15] M. Gromov, Hyperbolic groups, Essays in group theory, Math. Sci. Res. Inst. Publ., 8, pp. 75-263, Springer, New York, 1987.

[16] V. Guirardel and A. Ivanov, Non-nesting actions of Polish groups on real trees, J. Pure Appl. Algebra 214 (2010), no. 11, 2074-2077.

[17] M. Hamann, On the tree-likeness of hyperbolic spaces, preprint.

[18] B. Hughes, Trees and ultrametric spaces: a categorical equivalence, Adv. Math. 189 (2004), no. 1, 148-191.

[19] W. Imrich, On metric properties of tree-like spaces, Contributions to graph theory and its applications (Internat. Colloq., Oberhof, 1977), pp. 129-156, Tech. Hochschule Ilmenau, Ilmenau, 1977.

[20] I. Kapovich and N. Benakli, Boundaries of hyperbolic groups, Combinatorial and geometric group theory, Contemp. Math., 296, pp. 39-93, Amer. Math. Soc., Providence, RI, 2002.

[21] I. Kapovich and M. Lustig, Stabilizers of R-trees with free isometric actions of $F_{N}$, J. Group Theory 14 (2011), no. 5, 673-694.

[22] W. A. Kirk, Hyperconvexity of R-trees, Fund. Math. 156 (1998), no. 1, 67-72.

[23] _ Fixed point theorems in CAT(0) spaces and R-trees, Fixed Point Theory Appl. 4 (2004), 309-316. 
[24] G. Levitt, Non-nesting actions on real trees, Bull. Lond. Math. Soc. 30 (1998), no. 1, 46-54.

[25] J. C. Mayer, L. K. Mohler, L. G. Oversteegen, and E. D. Tymchatyn, Characterization of separable metric R-trees, Proc. Amer. Math. Soc. 115 (1992), no. 1, 257-264.

[26] J. C. Mayer, J. Nikiel, and L. G. Oversteegen, Universal spaces for R-trees, Trans. Amer. Math. Soc. 334 (1992), no. 1, 411-432.

[27] J. C. Mayer and L. G. Oversteegen, A topological characterization of R-trees, Trans. Amer. Math. Soc. 320 (1990), no. 1, 395-415.

[28] J. R. Munkres, Topology: a first course, Prentice-Hall, Inc., Englewood Cliffs, NJ, 1975.

[29] F. Paulin, The Gromov topology on R-trees, Topology Appl. 32 (1989), no. 3, 197221.

[30] K. Ruane, CAT(0) groups with specified boundary, Algebr. Geom. Topol. 6 (2006), 633-649.

Department of Mathematics and

Statistics

Mississippi State University

Drawer MW

Mississippi State, MS 39762

USA

pfpoke@gmail.com 